\title{
Effects of Cathode Configuration on Hall Thruster Cluster Plume Properties
}

\author{
Brian E. Beal* and Alec D. Gallimore \\ University of Michigan, Ann Arbor, Michigan 48109 \\ and \\ William A. Hargus Jr. \pm \\ U.S. Air Force Research Laboratory, Edwards Air Force Base, California 93524 \\ DOI: $10.2514 / 1.24636$
}

\begin{abstract}
Clusters of Hall thrusters may be used to produce electric propulsion systems capable of operating at power levels in excess of the current state of the art. One of the key factors to be considered in determining the optimum cluster architecture is the configuration of the electron-emitting cathode(s). This work presents experimentally determined plume properties and discharge current characteristics obtained with multiple thrusters coupled to a single cathode. Spatially resolved plasma density, electron temperature, and plasma potential data are presented during both singlethruster and cluster operation. Measurements taken in this configuration are compared with previously published data obtained with each thruster coupled to its own independent cathode. Critical plasma parameters in the cluster plume are shown to be strongly influenced by the location of the hollow cathode.
\end{abstract}

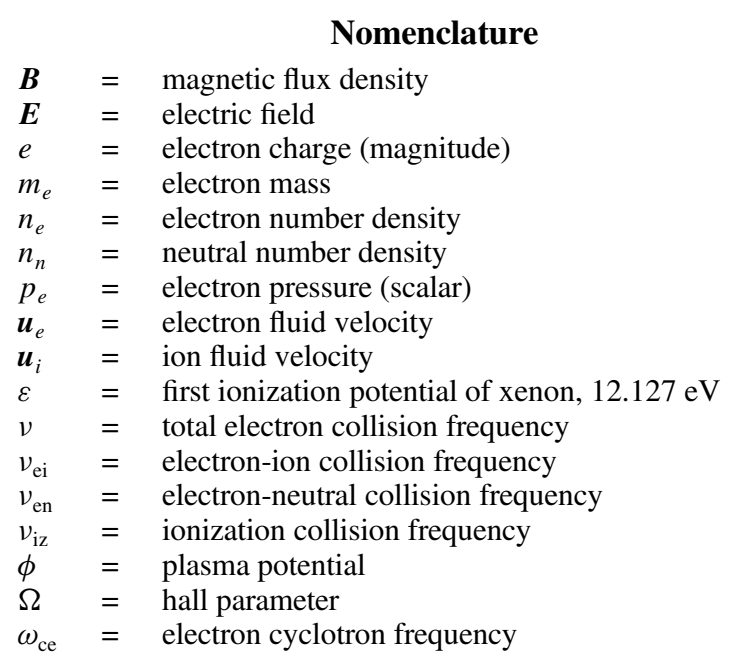

Subscripts

$\begin{aligned} R & =\text { radial direction } \\ Z & =\text { axial direction } \\ \theta & =\text { azimuthal direction }\end{aligned}$

\section{Introduction}

$\mathbf{M}$ ANY future spacecraft will use electric propulsion systems for stationkeeping, rephasing, and orbit topping applications, as well as for deep-space missions. Because of its combination of high reliability and high thrust density at moderately high specific impulses, the Hall thruster is particularly well suited to many of these

Presented as Paper 3678 at the 41st AIAA/ASME/SAE/ASEE Joint Propulsion Conference and Exhibit, Tucson, AZ, 10-13 July 2005; received 15 April 2006; revision received 29 November 2006; accepted for publication 3 December 2006. Copyright (C) 2007 by the American Institute of Aeronautics and Astronautics, Inc. All rights reserved. Copies of this paper may be made for personal or internal use, on condition that the copier pay the $\$ 10.00$ per-copy fee to the Copyright Clearance Center, Inc., 222 Rosewood Drive, Danvers, MA 01923; include the code 0748-4658/07 \$10.00 in correspondence with the CCC.

*Plasmadynamics and Electric Propulsion Laboratory, currently U.S. Air Force Research Laboratory, Edwards Air Force Base, CA 93524; brian.beal@edwards.af.mil.

${ }^{\dagger}$ Spacecraft Propulsion Branch. missions. The Hall thruster is an annular device in which a propellant, usually xenon, is ionized and then accelerated by electrostatic forces to create propulsive thrust. In this type of device, electrons from a thermionically emitting hollow cathode proceed upstream toward a positively biased anode in which they ionize the injected propellant. A radial magnetic field imposed by an electromagnetic circuit impedes the motion of electrons toward the anode. The magnetic field strength is such that the electron gyroradius is much smaller than the characteristic dimensions of the device, whereas the ion gyroradius is much larger. This arrangement facilitates a strong axial electric field within the plasma and provides for acceleration of the positively charged xenon ions. Upon exiting the device, the ion beam is neutralized by electrons from the hollow cathode, thus maintaining quasi neutrality within the plasma plume. The crossed electric and magnetic fields cause electrons in the discharge channel to drift azimuthally, thereby creating a closed-drift electron Hall current from which this type of thruster derives its name. Further information on the general characteristics of Hall thrusters can be found in numerous publications [1,2].

One method being considered for reaching the increasing power levels required for future applications involves clustering multiple devices of moderate power to reach the total throughput required $[\underline{3}, \underline{4}]$. The clustered approach offers several advantages over using a single monolithic thruster, including improved system reliability, modularity, and the ability to throttle the system by simply turning on or off the appropriate number of thrusters. Throttling the system in this way allows the cluster to operate at various powers without running any individual thruster at off-design conditions and may prove beneficial for missions in which either the propulsive needs or the available power vary with time.

Although using a cluster of high-power thrusters for primary propulsion appears to be advantageous for many missions, there are several systems integration issues that must be considered before clusters can be used in flight $[3,4]$. For example, it is imperative that the interaction of the plasma plumes, both among the thrusters and with the spacecraft, be understood. In an effort to address this issue, a cluster of four Busek BHT-200-X3 200-W class devices has been studied in detail and reported on previously [5-9]. In these studies, plasma properties such as electron number density, electron temperature, and plasma potential were measured downstream of a cluster and compared with properties measured downstream of a single thruster. This work demonstrated the methods by which knowledge of plasma parameters downstream of a single thruster can be used to accurately predict critical plasma parameters downstream 
of a multithruster array when each thruster is operated independently; that is, with its own dedicated hollow cathode and power circuit $[8,9]$. In this configuration, analytical methods were shown to be capable of predicting the electron number density, electron temperature, and plasma potential in a cluster plume to within the margin of error of typical plasma diagnostics.

Although the nominal (i.e., independent) cluster configuration considered previously may be preferred in many cases, due to its favorable combination of modularity and scalability, there are some situations in which trade studies may show alternative cluster configurations to be advantageous. For example, it may be beneficial in some situations to operate a cluster of thrusters in parallel so that the entire assembly may be powered from a single large powerprocessing unit (PPU) rather than several smaller ones. In other situations, performance benefits may be achieved by operating multiple thrusters from a single cathode. Because propellant injected through the hollow cathode is not accelerated through the engine, it provides no thrust and therefore reduces the overall specific impulse of the system. Clearly, operating multiple thrusters from a single cathode (without increasing the cathode mass flow rate or with an increase that is less than linear with emitted current) would mitigate the effects of this loss mechanism, compared with operating each thruster with its own cathode. Although reliability considerations almost certainly eliminate the possibility of using only a single cathode with an entire multithruster array in an operational cluster design, one can envision the use of a single cathode with one or more backup units for the entire cluster or a reconfigurable system that could support shared-cathode operation in the event of a single-unit failure. The two latter configurations would provide significant risk reduction for spacecraft designers. This paper examines some of the technical issues and challenges related to each of these alternative configurations.

\section{Experimental Apparatus}

\section{Cluster}

The cluster used in this experiment was composed of four Busek BHT-200-X3 200-W class Hall thrusters. An earlier version of this thruster was reported to operate at an anode efficiency of $42 \%$ and specific impulse of $1300 \mathrm{~s}$ while providing $12.4 \mathrm{mN}$ of thrust at the nominal operating conditions [10]. Each thruster had a mean discharge channel diameter of $21 \mathrm{~mm}$ and was operated on xenon propellant. The thrusters were arranged in a $2 \times 2$ grid with approximately $11.4 \mathrm{~cm}$ between the centerlines of nearest neighbors. Typical operating conditions for the BHT-200 are given in Table 1.

The naming convention and coordinate system used throughout this experiment are shown in Fig. 1. As shown, the thrusters were labeled as TH 1-4 beginning in the upper left-hand corner and proceeding counterclockwise when viewed from downstream. The origin of the coordinate system was defined as the midpoint of the cluster in the displayed $X-Y$ plane. The $Z$ coordinate measured the distance downstream of the thruster exit plane. A three-dimensional positioning system was used to sweep probes through the plasma plume.

Several different experimental configurations were tested to explore the various modes of cluster operation discussed in the previous section. In the first arrangement, both thrusters 2 and 3 were operated in parallel from a single discharge power supply. The main

Table 1 Typical operating conditions for the BHT-200 Hall thruster

\begin{tabular}{lc}
\hline \hline Parameter & Value \\
\hline Discharge voltage, V & $250 \pm 0.5$ \\
Discharge current, A & $0.80 \pm 0.03$ \\
Cathode potential, V & $-8.5 \pm 1.0$ \\
Electromagnet current, A & $1.0 \pm 0.03$ \\
Keeper current, A & $0.5 \pm 0.05$ \\
Keeper voltage, V & $13 \pm 1$ \\
Anode mass flow rate, sccm & $8.5 \pm 0.85$ \\
Cathode mass flow rate, sccm & $1.0 \pm 0.1$ \\
\hline \hline
\end{tabular}

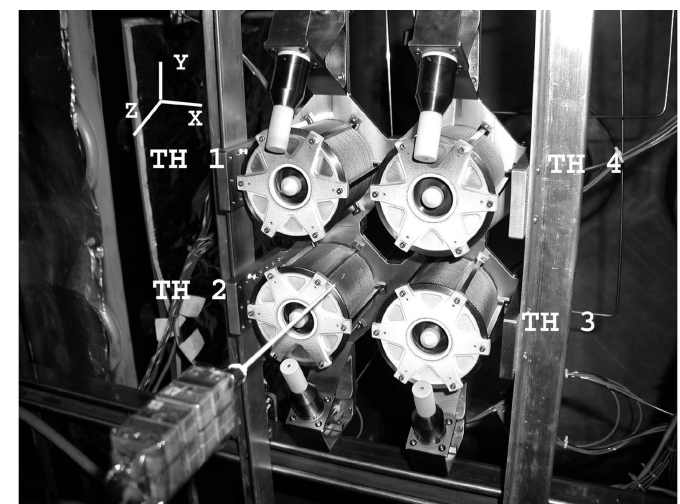

Fig. 1 The coordinate system and thruster naming convention used throughout this paper. For some tests, an additional cathode (not shown) was placed at the geometric center of the cluster.

goal of operating the thrusters in parallel was to examine the possibility of cathode current sharing between the devices through the plasma plume. The electromagnet, keeper, and cathode heater circuits remained separate between the thrusters. The current emitted by each cathode was measured using powered Hall effect sensors.

In the second experimental configuration, two thrusters were operated from a single hollow cathode to examine the effects of cathode number and placement on plume properties. This was accomplished with two separate cathode arrangements. In one case, two thrusters were operated from cathode 3. Measurements were conducted in chamber 6 (described next) with thrusters 3 and 4 operating from cathode 3 , which is a 3.2-mm-diam device. Sharedcathode tests in the large vacuum test facility (LVTF), which is described next, used thrusters 2 and 3 . The choice of different thrusters was made to accommodate the different probe positioning stages available in each facility. In both facilities, the xenon flow rate through the cathode remained constant at $1 \mathrm{sccm}$. The second neutralizer tested in this "shared-cathode" configuration was a 6.4mm-diam model HCN-252 hollow cathode available from Ion Tech, Inc. It was placed at the center of the cluster and operated at its nominal xenon flow rate of $5 \mathrm{sccm}$.

\section{Vacuum Facilities}

Two different vacuum facilities were used for various portions of the tests described here. The first was the LVTF at the University of Michigan. The LVTF is a stainless-steel-clad, cryopumped chamber that is $6 \mathrm{~m}$ in diameter, 9-m long, and is described in detail elsewhere [8]. The LVTF features a maximum pumping speed of $240,000 \mathrm{l} / \mathrm{s}$ on xenon and achieves a typical base pressure of approximately $1.5 \times 10^{-7}$ torr. For the tests reported here, only four of the seven available cryopumps were used, resulting in chamber background pressures ranging from $1.1 \times 10^{-6}$ torr for single-thruster operation to $3.6 \times 10^{-6}$ torr (corrected for xenon) during operation of all four thrusters.

The second vacuum facility used in these experiments was chamber 6 at the U.S. Air Force Research Laboratory. Chamber 6 is a $1.8 \times 3.0 \mathrm{~m}$ cylindrical, stainless steel vacuum chamber that is evacuated by one dual-stage cryopump and four single-stage cryopanels. During thruster operation, the chamber pressure stabilized at approximately $6.1 \times 10^{-6}$ torr for single-thruster operation and $2.3 \times 10^{-5}$ torr for four-thruster operation. Both reported pressures are corrected for xenon.

\section{Triple Probe}

A symmetric triple Langmuir probe was used to acquire spatially resolved measurements of plasma density and electron temperature throughout the cluster plume. This probe consisted of three tungsten electrodes insulated from each other by an alumina rod. The exposed section of each electrode was $5.0-\mathrm{mm}$ long and $0.5 \mathrm{~mm}$ in diameter. The electrodes were spaced approximately two electrode diameters 
apart, and the probe was sized to criteria that allowed the standard thin-sheath assumptions of probe theory to be applied [11].

The methods used to determine electron temperature and plasma density from raw triple probe data have been presented in detail elsewhere $[\underline{8}, \underline{9}, \underline{12}]$. Various previously published error analyses indicate that the absolute uncertainties in the calculated electron temperature and plasma density for typical triple probes are less than 30 and $60 \%$, respectively $[13,14]$. The relative uncertainty between multiple data points measured using the same probe is believed to be considerably less than the absolute uncertainty, due to the fact that many sources of error (uncertainty in probe dimensions, slight asymmetry of the electrodes, etc.) remain constant over the entire spatial region.

\section{Emissive Probe}

Plasma potential measurements were conducted using a floating emissive probe similar to the one described by Haas and Gallimore [15]. The emitting portion of the probe consisted of a loop of 0.13mm-diam tungsten filament, the ends of which were inserted into double-bore alumina tubing, and 0.51-mm-diam molybdenum wire leads. Short lengths of tungsten wire were inserted into the alumina tube to insure contact between the emitting filament and molybdenum leads. The diameter of the emitting filament loop was approximately $3 \mathrm{~mm}$. The normal to the plane of the loop formed by the emitting filament was oriented in the $X$ direction, shown in Fig. 1 .

The emissive probe is a widely used plasma diagnostic for which the operation is based on the premise that a thermionically emitting filament in a low-temperature plasma will approach the local plasma potential when its emitted electron current is sufficient to neutralize the plasma sheath [16]. In actuality, the floating potential of the emissive probe remains slightly below the true plasma potential, due to space-charge saturation of the sheath. For heavy ions, such as xenon, Ye and Takamura [17] showed that the difference between the probe potential and the true plasma potential can be as much as 1.03 times the local electron temperature (in eV). In the far field of the Hall thruster $(Z \geq 50 \mathrm{~mm})$, the error induced by this mechanism is less than $3 \mathrm{~V}$. For this experiment, the current necessary to heat the probe was provided by a programmable power supply with floating outputs. At each location in the plume, the current was steadily increased and the potential with respect to chamber ground at the negative terminal of the power supply was recorded. This method allowed for verification of a well-defined plateau in the voltagecurrent trace, indicating saturation of the plasma sheath. Considering that the voltage drop across the emitting filament never exceeded $6 \mathrm{~V}$, the potential was measured at the negative terminal of the probe, and the electron temperature over the majority of the plume was less than $3 \mathrm{eV}$, the absolute uncertainty in the plasma potential measurements is estimated to be -3 and $+8 \mathrm{~V}$. The relative uncertainty between data points obtained using the same probe is believed to be significantly smaller than these values, because the main source of uncertainty, the $\sim 5 \mathrm{~V}$ potential difference across the emitting filament, remained essentially constant over the entire sampled range. The relative uncertainty between data points is therefore conservatively estimated to be $\pm 2.0 \mathrm{~V}$ and is dominated by variations in electron temperature that can influence the small potential drop across the sheath surrounding the emitting filament.

\section{Results \\ Discharge Current Characteristics \\ Discharge current levels recorded with two thrusters (TH2 and TH3) operating in parallel are shown in Table 2. As shown, the current flowing through each anode is approximately $0.80 \mathrm{~A}$ and is nearly constant between the thrusters. This result is to be expected, because the anode current is controlled primarily by the propellant mass flow rate through each engine. The cathode current levels, on the other hand, show distinct differences between the two units.}

${ }^{\ddagger}$ Each keeper was operated at $0.5 \mathrm{~A}$, which is not included in the values shown in Table 2.
Table 2 Anode and cathode currents observed during parallel operation

\begin{tabular}{lc}
\hline \hline Electrode & Current, A \\
\hline Anode 2 & 0.80 \\
Anode 3 & 0.75 \\
Cathode 2 & -0.09 \\
Cathode 3 & 1.66 \\
\hline \hline
\end{tabular}

Table 2 shows that cathode 3 supplied essentially all of the current necessary to operate both engines despite the fact that both cathodes were operated at identical mass flow rates and keeper currents. The reason for this imbalance is not fully understood, but it may be related to slight differences in the effective work functions of the cathodes, due to differences in cumulative run times or manufacturing tolerances.

The dominance of one cathode shown in Table $\underline{2}$ has potentially important implications for Hall thruster cluster design. In particular, it implies that thruster-cathode pairs intended for parallel operation will likely require active current-balancing circuitry in the PPU to prevent one cathode from emitting more than the intended fraction of electron current. Similarly, any attempts to operate a single highcurrent thruster by supplying electron current from multiple lowcurrent cathodes are likely to be unsuccessful unless precautions are taken to ensure equal loading between the emitters.

In the second cluster configuration studied, two Hall thrusters were coupled to a single shared cathode. Although no qualitative changes in discharge current characteristics were noted when two thrusters were coupled to a single cathode, distinct differences in operating characteristics were observed when a single thruster was operated from a distant cathode. The discharge current and cathode potential data displayed in Fig. $\underline{2}$ were obtained with both $\mathrm{TH} 2$ and TH3 coupled to cathode 3 in the LVTF. As shown, when TH2 was operated alone with cathode 3 (i.e., from a time of 0 to approximately 2300_s), the discharge current was slightly higher than the nominal value of $0.80 \mathrm{~A}$, and the amplitude of current oscillations was also higher than observed in the nominal configuration [8]. When TH3 was ignited (a time of approximately $2300 \mathrm{~s}$ ), the discharge current and magnitude of oscillations in $\mathrm{TH} 2$ decreased to near nominal levels. At the same time, the cathode potential increased (moved closer to ground) by about $2.5 \mathrm{~V}$, thus bringing it to near the nominal level [8]. When TH3 was then shut off (a time of approximately $3200 \mathrm{~s})$ without changing any settings to $\mathrm{TH} 2$, the discharge current and cathode potential returned to their original anomalous values.

\section{Plasma Density}

The triple probe was used to measure the plasma density in the plume for both shared-cathode configurations: with the large center cathode shared and with cathode 3 shared. Measurements were obtained in chamber 6 with TH3 and TH4 operating individually and simultaneously. Figures 3 shows the profiles recorded at a selected axial location in the plume (50 $\mathrm{mm}$ downstream). Figure $3 \mathrm{a}$ depicts the density profile recorded with two thrusters coupled to the cathode

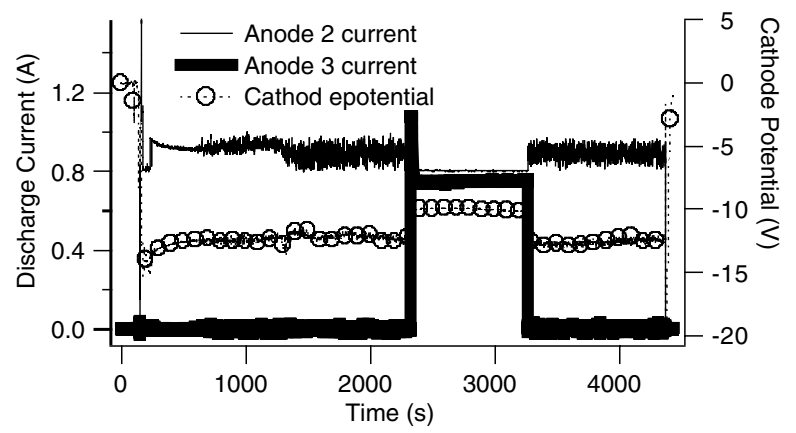

Fig. 2 Operating characteristics of two Hall thrusters coupled to a single hollow cathode. 


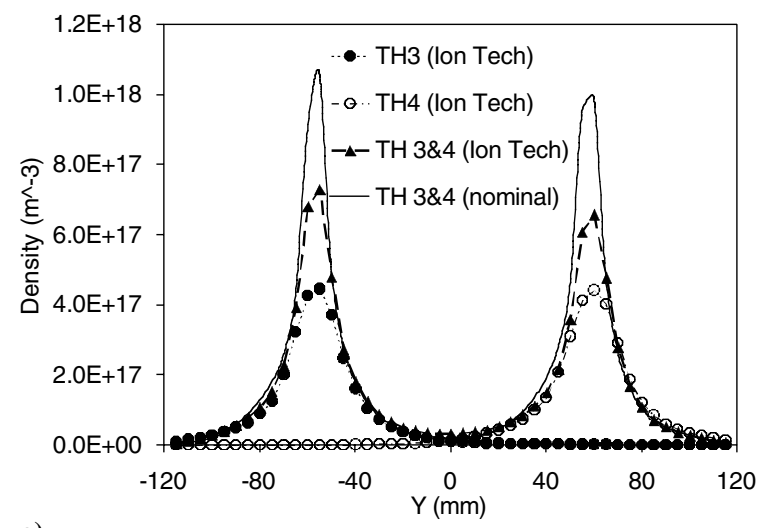

a)

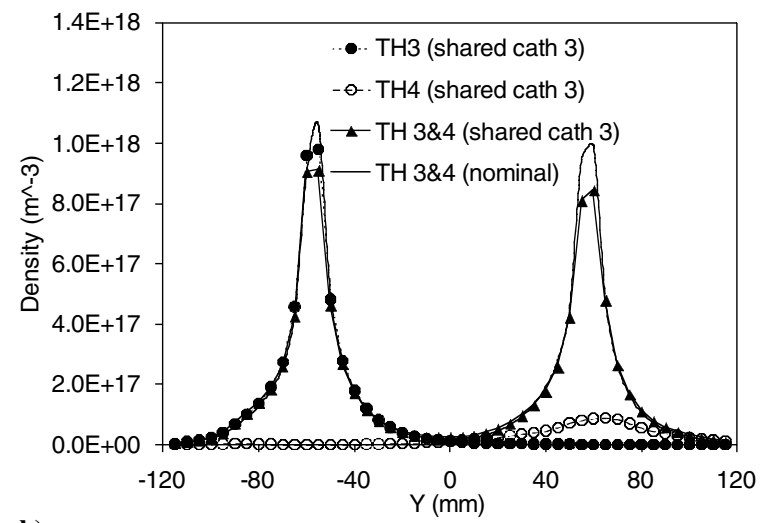

b)

Fig. 3 Density profiles recorded $50 \mathrm{~mm}$ downstream of TH 3 and 4 during operation with a) a shared cathode in the center of the cluster and b) cathode 3 shared.

in the center of the cluster, and Fig. $3 \mathrm{~b}$ reflects the results of coupling to cathode 3 . The thick black line in each figure depicts the density profile measured with each thruster operating in conjunction with its own cathode; that is, in the nominal configuration that was reported on previously $[8,9]$. Note that additional measurements taken in the same configuration reveal the same trends discussed here and have been presented elsewhere $[8,12]$.

The plasma density measurements shown in Fig. 3 reveal several interesting features related to shared-cathode operation. First, the density downstream of a cluster operating with a single neutralizer cannot be predicted by simply summing the contributions from each individual thruster, as they can in the completely modular configuration [8,9]. This finding is particularly evident from examination of the data taken with cathode 3 shared. In this situation, TH3 shows no unusual plume characteristics when operating alone, which is to be expected, because it is coupled to its own cathode. When TH4 is operated from this same cathode, however, the plume appears very diffuse and the peak density is more than a factor of 10 lower than the one measured with the engine coupled to its own cathode. Most surprising is that the density downstream of TH4 increases to near the nominal profile (within about 25\%) when TH 3 and 4 are operated simultaneously. Clearly, operating both thrusters together changes the basic operation of TH4, thus eliminating the possibility of predicting the cluster plume via superposition. Incidentally, the data presented here suggest that it is the location of the hollow cathode and not the specific design of the electron emitter that causes changes in the plume properties. This observation is based on the fact that the profile downstream of TH4 differs greatly from that of TH3 when each is operated individually with cathode 3 . Increasing the distance between the thruster and the neutralizer seems to decrease the plasma density in the plume dramatically.

Examination of the data taken with the thrusters coupled to the central Ion Tech cathode reveals similar trends to those discussed earlier. Because this cathode is significantly farther away from the anode of each thruster than the cathodes of the nominal configuration
( $\sim 81 \mathrm{~mm}$ vs $\sim 28 \mathrm{~mm}$ from the thruster centerlines), the lower density observed in the plume with each thruster running individually is consistent with the observations reported earlier. When both thrusters are operated together, the peak density downstream of each engine increases significantly, compared with the level measured during individual operation. The plasma density with both thrusters operating from the central cathode, however, falls short of the ones measured with cathode 3 shared, as well as with those measured in the nominal configuration.

Although Fig. 3 shows clearly that the location of the cathode has a significant effect on the properties in the plasma plume, it offers few clues as to why this is the case. To provide a more extensive database for studying possible causes, several additional sets of measurements were obtained in the LVTF with TH2 and TH3 coupled to cathode 3. The configurations tested include the following: 1) TH2 running alone, 2) TH2 running and propellant flowing through TH3 (without a discharge), 3) $\mathrm{TH} 2$ running with propellant flowing through $\mathrm{TH} 3$ and electromagnet 3 energized, and 4) TH2 and TH3 operating simultaneously from cathode 3 .

The plasma density profiles recorded at two different locations downstream of TH2 and TH3 are displayed in Fig. 4. As shown in these plots, operating $\mathrm{TH} 2$ alone with cathode 3 resulted in a very diffuse plume with a low plasma density, which is in agreement with the behavior discussed earlier. The addition of flow through TH3, and the concomitant increase in local pressure, caused the density in the plume to increase by about a factor of 2, although it remained far below the levels exhibited during normal operation. Energizing the electromagnet of TH3 had very little effect. Finally, igniting TH3 caused the plasma density downstream of both thrusters to increase dramatically to levels consistent with those reported previously for operation in the independent, modular configuration [ $[\underline{8}, \underline{9}]$.

\section{Electron Temperature}

The same triple probe used to obtain the density measurements presented in the previous section also measured the local electron temperature. Figure $\underline{5}$ shows the electron temperatures measured in

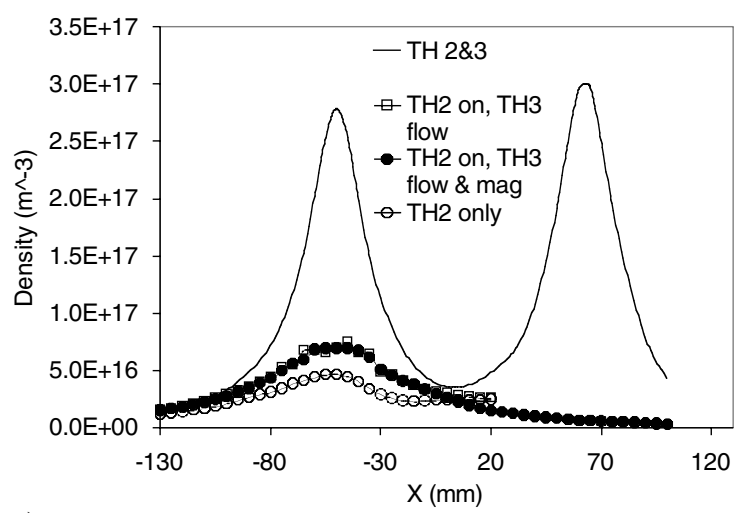

a)

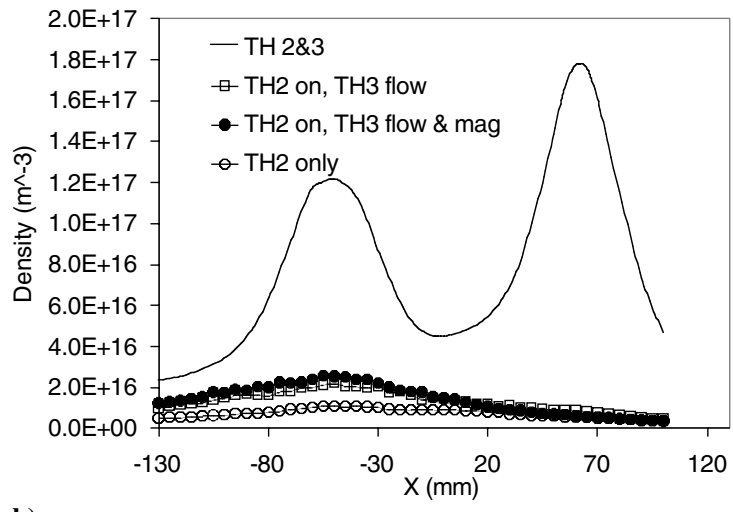

b)

Fig. 4 Density profiles recorded downstream of TH 2 and 3 during operation from cathode 3 at a) $Z=70 \mathrm{~mm}$ and b) $Z=120 \mathrm{~mm}$. 

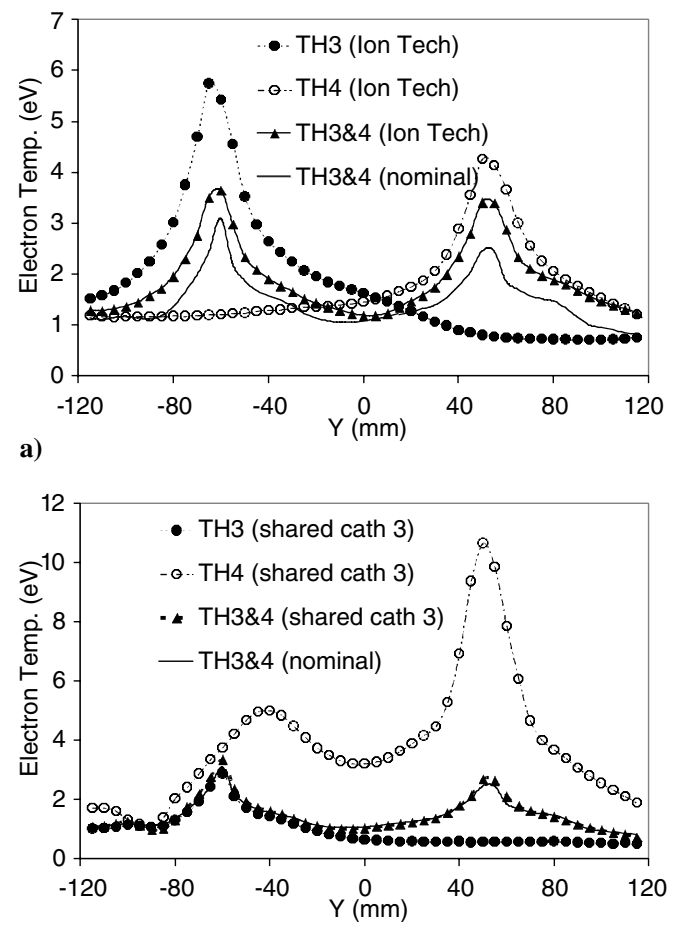

b)

Fig. 5 Electron temperature profiles recorded $50 \mathrm{~mm}$ downstream of TH 3 and 4 during operation with a) a shared cathode in the center of the cluster and b) cathode 3 shared.

chamber 6 for the two different shared-cathode experiments. As shown, the electron temperature downstream of a thruster tended to increase when it was operated with a distant cathode. For example, Fig. $5 \mathrm{~b}$ shows that the temperature peaked at over $10 \mathrm{eV}$ when TH4 was operated in conjunction with cathode 3 , compared with approximately $3 \mathrm{eV}$ during operation with a normally positioned cathode $[8,9]$. Coupling to the cathode in the center of the cluster caused similar behavior, and the peak electron temperature with one engine running rose to approximately $6 \mathrm{eV}$, as shown in Fig. $5 \mathrm{a}$. As expected, the peak electron temperature decreased with increasing downstream distance [12]. Even at an axial distance of $150 \mathrm{~mm}$, or approximately seven thruster diameters, the temperature downstream of TH4 remained approximately a factor of 2 higher when operated from a distant cathode than from a local one. Regardless of which cathode was used, running multiple thrusters tended to reduce the electron temperature in the plume, bringing it closer to the normal level. Operating both thrusters in conjunction with cathode 3 caused the electron temperature to fall to almost exactly the nominal values, whereas it remained somewhat above normal during operation of the central cathode [8].

Electron temperatures measured at two axial locations in the LVTF, with TH 2 and TH3 sharing a single Busek cathode, are shown in Fig. 6. As expected from the measurements obtained in chamber 6, operating TH2 with the distant cathode 3 caused the electron temperature in the plume to rise well above the values measured in the nominal configuration [8]. In this mode, the temperature along the centerline of TH2 was approximately $6.5 \mathrm{eV}$ at $Z=70 \mathrm{~mm}$ and fell to less than $2.5 \mathrm{eV}$ by $170 \mathrm{~mm}$ downstream of the exit plane. When an $8.5 \mathrm{sccm}$ propellant flow was initiated through thruster 3 (without igniting a discharge), the electron temperature downstream of TH2 fell to about $3.5 \mathrm{eV}$ at $70 \mathrm{~mm}$ and $1.5 \mathrm{eV}$ by $170 \mathrm{~mm}$ downstream. This is similar to the behavior of the plasma density, which also showed significant changes when the average neutral density between the thruster and cathode was increased. Energizing the electromagnet of thruster 3 caused a very slight increase in electron temperature in the region near the cathode. When TH3 was operated in conjunction with $\mathrm{TH} 2$, the electron temperature fell to nominal levels and exhibited a high degree of symmetry between the plumes of the two engines, despite the fact that the hollow cathode was much closer to TH3 than it was to TH2 (approximately $28 \mathrm{~mm}$

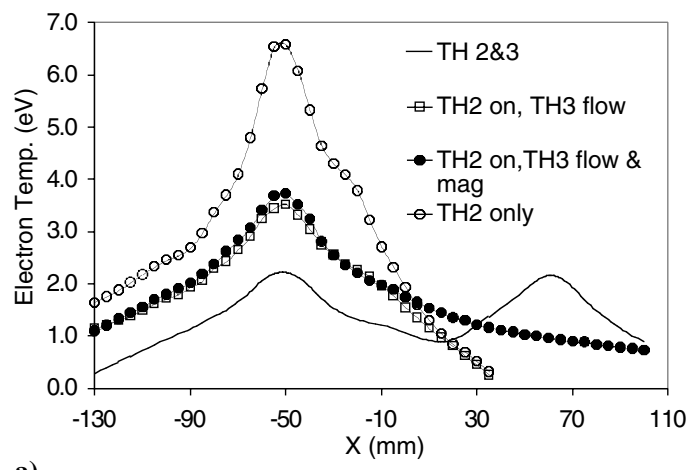

a)

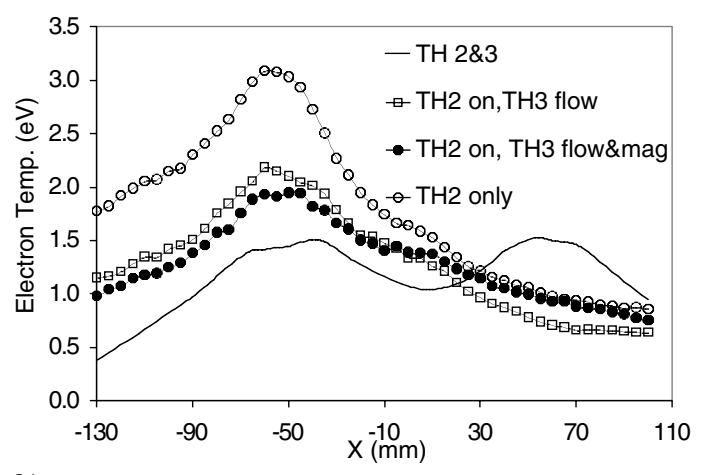

b)

Fig. 6 Electron temperature profiles recorded downstream of TH 2 and 3 during operation from cathode 3 at a) $Z=70 \mathrm{~mm}$ and b) $Z=120 \mathrm{~mm}$.

from the centerline vs $115 \mathrm{~mm}$ ). It can therefore be said that increasing the local pressure and igniting an intermediate thruster both tended to decrease the electron temperature in the plume of the thruster farthest from the cathode.

\section{Plasma Potential}

Like the plasma density and electron temperature, the plasma potential profiles in the plume also exhibited major changes from the nominal values (i.e., the values recorded when each thruster was operated with its own cathode) when the cluster was operated with a single shared cathode. Figure 7 shows potentials measured downstream of TH3 and TH4 for several different configurations at various axial positions. As shown, operating a single thruster from the 6.4-mm-diam cathode located at the center of the cluster caused the peak potential at $Z=50 \mathrm{~mm}$ to increase to more than $50 \mathrm{~V}$, compared with a nominal value of just over $20 \mathrm{~V}$ at this location. Operating both thrusters together with this cathode caused the peak plasma potential to fall to about $35 \mathrm{~V}$ at this location. Similar to the behavior observed in the profiles of number density and electron temperature, coupling two thrusters to a single cathode located in close proximity to one of the devices resulted in plasma potentials nearly identical to the ones recorded with each thruster operating independently. As expected, all of the potentials decreased with increasing axial distance. The relative positions of the curves, however, remained consistent, with the two-thruster, shared central cathode potentials falling between the nominal values and those measured with a single thruster operating from the central cathode.

Additional experiments were performed in the LVTF to examine the effects of neutral density and magnetic fields on the plasma potential profiles. Like the triple probe measurements, these data were recorded downstream of $\mathrm{TH} 2$ and $\mathrm{TH} 3$, with both devices tied to cathode 3 . The resulting data are presented in Fig. $\underline{8}$. The curves labeled "TH2 plus TH3 flow" represent data obtained with TH2 running and $8.5 \mathrm{sccm}$ of xenon flowing through $\mathrm{TH} 3$, whereas the flow through TH3 was increased to $17 \mathrm{sccm}$ for the curves labeled "TH2 plus TH3 double flow."

As shown in Fig. $\underline{8}$, the plasma potential downstream of TH2 was much higher at a given axial location when operated with cathode 3 


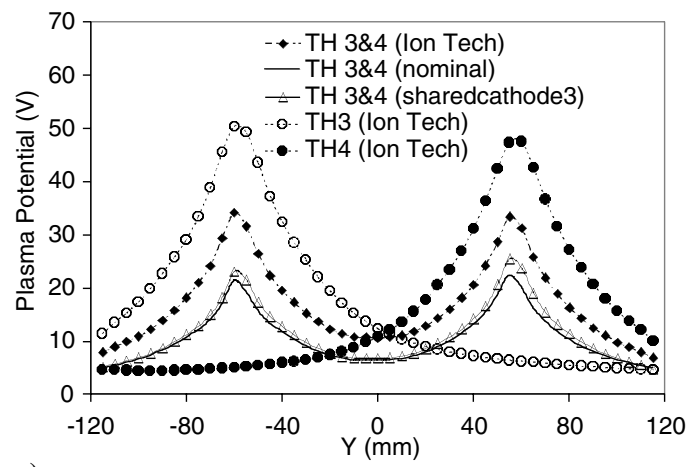

a)

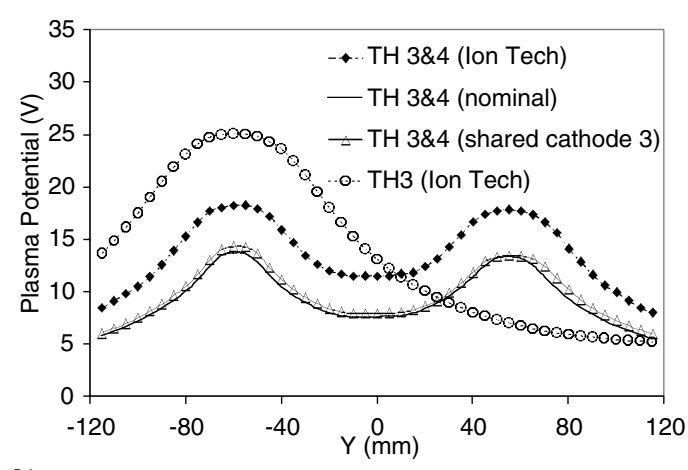

b)

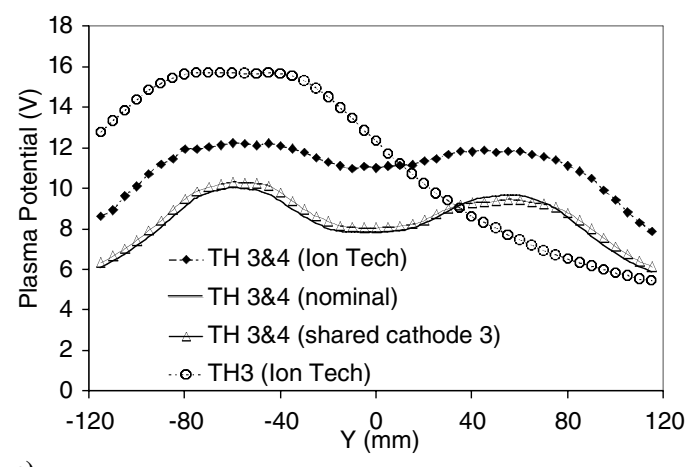

c)

Fig. 7 Plasma potential profiles measured downstream of TH 3 and 4 at a) $Z=50 \mathrm{~mm}$, b) $Z=100 \mathrm{~mm}$, and c) $Z=150 \mathrm{~mm}$ for various cathode configurations.

than it was in the nominal configuration presented previously [ $[\underline{8}, 9]$. Because the boundary conditions of the potential field were set by the applied discharge voltage, these measurements depict a "pushing out" of the plasma potential, such that a larger fraction of the potential drop occurred outside of the discharge channel. The larger potential drop outside of the engine may have a detrimental effect on thruster performance, because it is likely to lead to increased beam divergence. The bottom plots show that increasing the neutral density, and therefore the particle pressure, between the anode and the cathode reduced the potential in the plume somewhat. Finally, compared with the data measured with $8.5 \mathrm{sccm}$ flowing through TH3, energizing electromagnet 3 appeared to cause slight decreases in the plasma potential directly downstream of $\mathrm{TH} 2$ and increases in the potential directly downstream of the cathode. The magnitude of the change caused by the magnetic field, however, was relatively small, compared with the effect of adding flow. As expected, operating both thrusters together caused the potential in the plume to fall to almost exactly the values measured in the nominal configuration.

\section{Analysis}

The data presented in the previous sections indicate that the plasma plume properties and basic operating characteristics of a Hall

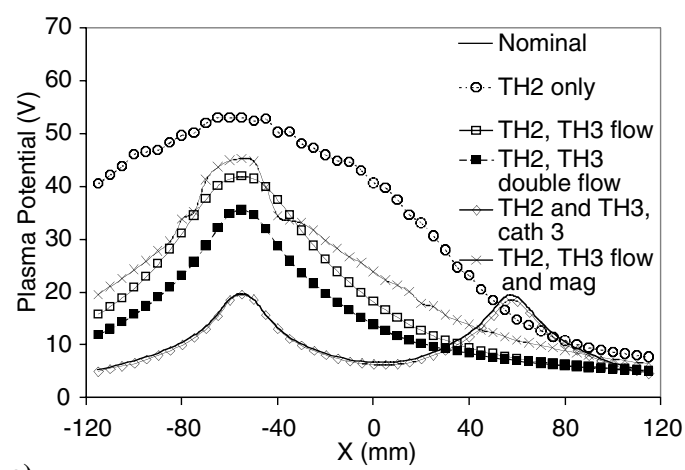

a)

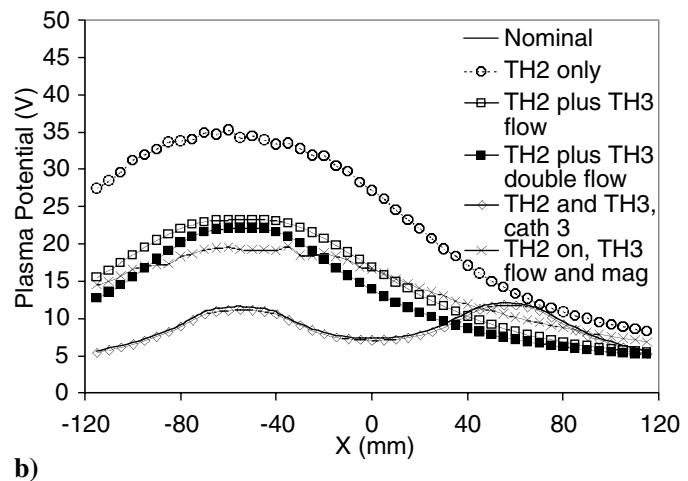

Fig. 8 Plasma potential profiles recorded downstream of TH 2 and 3 during operation from cathode 3 at a) $Z=70 \mathrm{~mm}$ and b) $Z=120 \mathrm{~mm}$.

thruster are both influenced by the coupling between the anode and cathode. The most important parameters controlling this process are likely to be the distance between the electrodes and the properties of the medium in the interelectrode gap. A qualitative understanding of the phenomena revealed here can be gained by considering which of several possible mechanisms dominate the coupling process. This is accomplished using the steady-state electron momentum equation given as Eq. (1), in which we have neglected electron inertia, assumed that the electron pressure tensor is isotropic, and taken the ratio of electron-to-ion density to be unity:

$$
\nabla \phi=-\boldsymbol{E}=\boldsymbol{u}_{e} \times \boldsymbol{B}+\frac{\nabla p_{e}}{n_{e} e}+\frac{m_{e}}{e}\left[\left(v_{\mathrm{en}}+v_{\mathrm{iz}}\right) \boldsymbol{u}_{e}+v_{\mathrm{ei}}\left(\boldsymbol{u}_{e}-\boldsymbol{u}_{i}\right)\right]
$$

The Hall thruster plume is often considered to be unmagnetized and nearly collisionless, such that the electron momentum equation may be reduced to Eq. (2):

$$
\nabla \phi=-\boldsymbol{E}=\frac{\nabla p_{e}}{n_{e} e}
$$

This equation, along with the experimentally measured electron temperature and number density, allows the ambipolar potential field to be predicted. This field is shown in Fig. 9, along with experimentally measured plasma potentials for $\mathrm{TH} 3$ operating from its own cathode and for $\mathrm{TH} 2$ operating from the distant cathode 3 . In each case, the radial coordinate denotes the distance from the operating thruster's centerline. The approximate radial location of the operating cathode is shown for each case.

As shown in Fig. 9, the ambipolar prediction is in excellent agreement with the measured profile in the region radially outboard of the cathode location for a thruster operating with its nominal (near) cathode and shows moderate divergence near the thruster centerline. The ambipolar plasma potential profile for operation from a distant cathode, however, diverges greatly from the measured profile, suggesting that one of the other terms in Eq. (1) is significant. The two possible sources to account for the existence of a nonambipolar electric field are the collisional term (related to classical resistivity) and the term involving the magnetic field (related to cross-field 


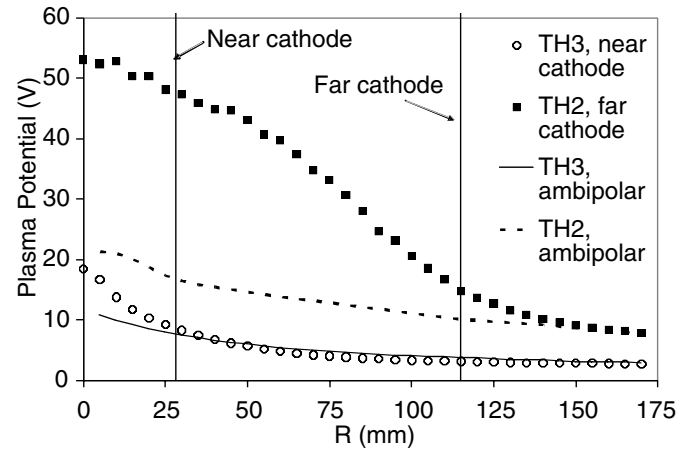

Fig. 9 Measured plasma potential profiles compared with those predicted from ambipolar expansion for thrusters operating with near and far cathodes at an axial distance of $70 \mathrm{~mm}$ downstream of the exit plane.

resistivity). The Hall parameter is a useful measure of the relative importance of the magnetic field to that of collisions and is given in Eq. (3), in which the total collision frequency is the sum of the electron-ion, electron-neutral, and ionization frequencies:

$$
\Omega \equiv \frac{\omega_{\mathrm{ce}}}{v}=\frac{e|\boldsymbol{B}|}{m_{e} v}
$$

An approximate value for the Hall parameter can be found by first calculating values of the relevant collision frequencies using Eqs. (46), in which the electron temperature is given in units of $\mathrm{eV} \mathrm{[18]:}$

$$
\begin{gathered}
v_{\mathrm{en}}=n_{n} \sqrt{\frac{8 e T_{e}}{\pi m_{e}}} \frac{\sqrt{\pi}}{2 \sqrt{2}} \frac{6.6 \cdot 10^{-19}\left(0.25 T_{e}-0.1\right)}{1+\left(0.25 T_{e}\right)^{1.6}} \\
v_{\mathrm{ei}}=2.9 \cdot 10^{-12} n_{e} T_{e}^{-3 / 2}\left[23-0.5 \ln \left(\frac{10^{-6} n_{e}}{T_{e}^{3}}\right)\right] \\
v_{\mathrm{iz}}=10^{-20} n_{n} \sqrt{\frac{8 e T_{e}}{\pi m_{e}}}\left(3.97+0.643 T_{e}\right) \exp \left[-\varepsilon / T_{e}\right]
\end{gathered}
$$

Relevant collision frequencies are displayed in Fig. 10, in which the neutral density has been assumed to be $5.2 \times 10^{16} \mathrm{~m}^{-3}$, which is consistent with the measured background pressure of $1.1 \times 10^{-6}$ torr [8]. These collision frequencies were used in conjunction with previously published magnetic field measurements to calculate the Hall parameters displayed in Fig. 11 [8]. The Hall parameter is shown to be in excess of 500 throughout the region of interest and approaches 2,000 as the displaced cathode is approached, which indicates that the effect of the magnetic field should dominate over collisional phenomena. That the Hall parameter takes such large values is somewhat unexpected, because the magnetic field, which is predominantly axial in this region of the plume, is only approximately 2-3 $\mathrm{G}$ in magnitude. However, the electron gyroradius in the vicinity of the cathode can be shown to be on the order of $10-15 \mathrm{~mm}$. This value is one order of magnitude smaller than the characteristic distance between the thruster and the displaced cathode, and roughly two orders of magnitude smaller than the local electron collision mean free path, both of which suggest that the magnetic field will influence the electron dynamics for the reported experimental conditions.

Further confidence that the effects discussed in this work are a result of cross-field electron transport can be gained by considering the trends that were observed when the local neutral density was increased by adding flow through the nonoperating thruster. Rewriting the radial component of Eq. (1) with the help of an alternate expression for the Hall parameter given as Eq. (7) leads to Eq. ( ), in which we have assumed the electron fluid velocity to be

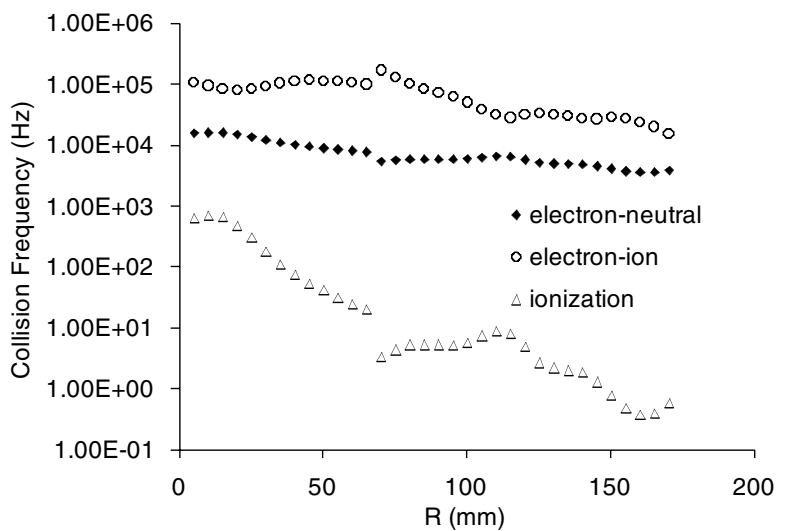

Fig. 10 Relevant collision frequencies at an axial distance of $70 \mathrm{~mm}$ during operation from a distant cathode. The apparently large jump at approximately $R=70 \mathrm{~mm}$ is an artifact of a single anomalous electron temperature reading and does not represent a true singularity in the data.

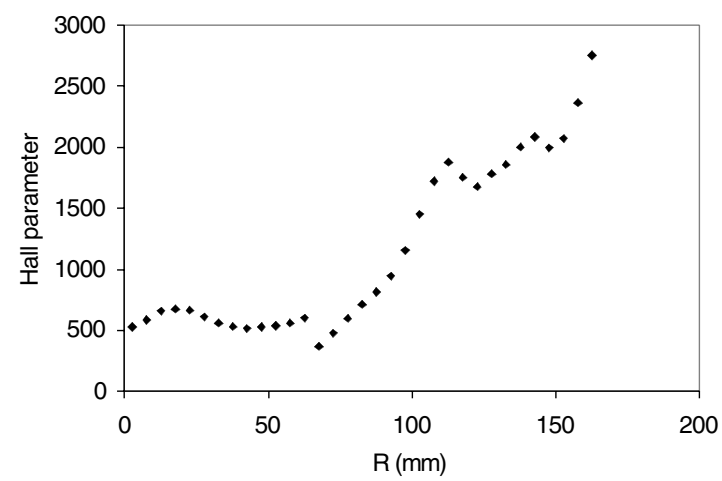

Fig. 11 Calculated Hall parameters as a function of radial location at $Z=70 \mathrm{~mm}$ during operation from a distant cathode. Note the large values despite the low magnetic field strength in the far-field plume. The apparently large jump at approximately $R=70 \mathrm{~mm}$ is an artifact of a single anomalous electron temperature reading and does not represent a true singularity in the data.

much greater than the ion fluid velocity in the region of interest [19]:

$$
\begin{gathered}
\Omega \approx \frac{u_{e, \theta}}{u_{e, r}} \\
\frac{\partial \phi}{\partial r}=-E_{r}=\frac{\partial p_{e}}{\partial r} \frac{1}{e n_{e}}+\Omega B_{z} u_{e, r}+\frac{m_{e} v}{e} u_{e, r}
\end{gathered}
$$

When the neutral density was increased by adding flow through the nonoperating thruster, all three electron collision rates of interest increased. The ionization and electron-neutral collision rates increased proportionally to the increase in neutral density, according to Eqs. (4) and (6), whereas the electron-ion collision rate increased as a result of the increased plasma density induced by ionization of a portion of the injected gas. If cross-field electron transport were a dominant mechanism, the increased collision rate would lower the Hall parameter and therefore decrease the magnitude of the second term on the right-hand side (RHS) of Eq. (8), which would, in turn, decrease the plasma potential at a given location in the far-field plume. This trend is exactly the one that was observed. On the other hand, if cross-field transport were negligible and the nonambipolar contribution to the electric field were a result of interparticle collisions, increasing the neutral density should have increased the magnitude of the third term on the RHS of Eq. ( 1 ) and therefore caused an upward shift in the plasma potential profile. That this was not observed supports our assertion that cross-field mobility 


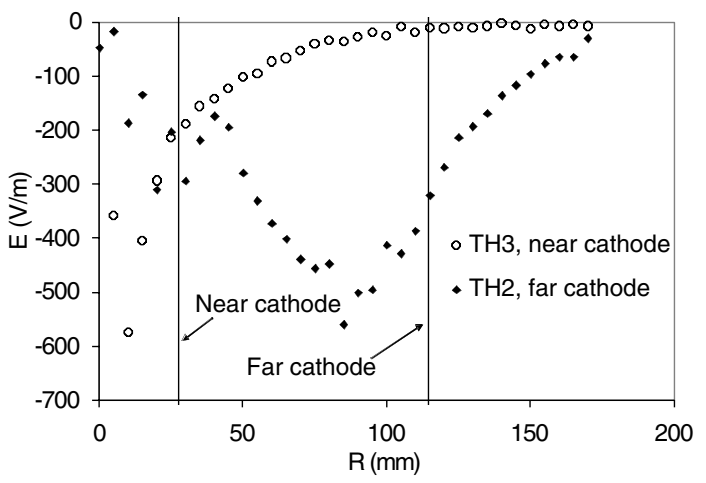

Fig. 12 Radial electric fields $70 \mathrm{~mm}$ downstream of the thruster exit plane for thrusters operating with both near and far cathodes.

dominates the cathode coupling process for the experimental conditions reported here.

Two additional trends revealed by this study are the behavior of the electron temperature profiles when the neutral density was increased and the behavior of the potential profiles when the adjacent magnet was turned on. First, the electron temperature trends can be understood by noting that the main source of electron heating in the plume is the electric field, which transfers energy to electrons at the rate of $\boldsymbol{j}_{e} \cdot \boldsymbol{E}$. The main energy loss mechanism is ionization [18]. The effect of increasing the neutral density is then twofold: it decreased electron heating by reducing the magnitude of the electric field in the far field, and it increased electron cooling by increasing the ionization collision frequency. Both of these effects tended to lower the observed electron temperatures.

Unlike the effect of adding flow through the nonoperating thruster, activating its electromagnet had relatively little effect on the plume properties. This is explained by examining previously published magnetic field profiles, which show only a modest change in the field strength in the immediate vicinity of the cathode when this magnet was activated, compared with when it was not [8]. In fact, activating this second electromagnet caused only about a $1 \mathrm{G}$ increase in the axial field strength near the cathode and a negligible change over a large fraction of the plume. This change then could be expected to show modest increases in plasma potential in the vicinity of the cathode, but only very small changes elsewhere. Close examination of Fig. 8a reveals that this is precisely what happened. Further insight into the small magnitude of this change is gained by examining Fig. 12, which shows the electric field strengths calculated from the plasma potential profiles recorded during operation from both a near cathode and a distant one. Note the similarity between the peak radial electric field strengths recorded for each case. The large changes in plasma potential noted during operation from a distant cathode are the result of integrating a small nonambipolar electric field over a large distance, as opposed to a significant increase in the peak electric field strength itself. This further explains why the small change in electric field strength induced by activation of the second electromagnet had a much smaller effect on the plasma potential than did changing the location of the cathode.

One final observation is an apparent discrepancy between the results presented here and those of Walker [20] and Zakharenkov, et al. [21], who found that Hall thrusters could be operated with cathodes placed several thruster diameters away with no apparent effect on performance. There are at least three possibilities that may be considered to explain this. First, because thrust was not measured as part of the present investigation, one could hypothesize that the changes in plume properties discussed earlier occurred without being accompanied by a change in performance. We view this hypothesis as being very unlikely. Second, because both Walker and Zakharenkov, et al. studied larger thrusters, $\underline{s}$ it might be reasonable to suppose that larger thrusters are in some way less sensitive to cathode location than the $200-\mathrm{W}$ engines studied here. Third, it is possible to

${ }^{\S}$ Walker used the 5-kW P5 thruster, Zakharenkov, et al. used a cluster of three 1.5-kW D-55 anode layer thrusters. hypothesize that there may be a certain design feature (not related to power level) that makes particular thrusters more or less sensitive to cathode position. The most likely cause is a combination of the second and third points. First, because both of the other studies concentrated on larger thrusters, both were conducted at higher background pressures of 5-9 $\times 10^{-6}$ torr in one case [20] and more than $1 \times 10^{-4}$ torr in the other [21]. The higher pressure would be expected to reduce the Hall parameter and therefore the importance of cross-field mobility in the case of the test conducted on the D-55 thrusters [21]. Because the study of the P-5 cluster was conducted at a similar pressure to the present study, however, it is doubtful that pressure effects alone accounted for the different sensitivities to cathode location. Second, because it has been shown that magnetic fields of only a few gauss can affect transport in the far field, it is possible that slight differences in the fringing fields from different thrusters could have a significant effect on plume properties. It is unclear from the available data, however, whether variations between the magnetic fields of the various thrusters contributed to the disparate results.

\section{Conclusions}

An extensive array of thruster operating parameters and plasma plume properties have been measured for clusters operating in both a parallel configuration and with multiple thrusters coupled to a single cathode. The results show that parallel operation tends to allow one cathode to dominate the discharge by emitting the majority of the required electron current. When multiple thrusters are operated in conjunction with a single cathode, however, plume measurements show pronounced differences in plume properties, depending on the number of thrusters in operation. In particular, operating a thruster from a distant cathode rather than a local one has been shown to cause increases in plasma potential and electron temperature, as well as a decrease in plasma density, in the near-field plume. When multiple thrusters were operated with a single cathode, the key plume parameters returned to near nominal levels. The dependence of the basic operating properties of any given thruster on the characteristics of adjacent units makes the shared-cathode cluster configuration an unlikely choice for operational spacecraft. The nominal cluster configuration in which each thruster is operated with its own independent cathode is likely to be the most beneficial approach for development of high-power clusters.

\section{Acknowledgments}

Portions of this work were performed under the auspices of the U.S. Air Force Office of Scientific Research grant F49620-02-10051 and U.S. Air Force Research Laboratory/ERC, Inc. grant RP020870. The authors wish to thank Mitat Birkan of the U.S. Air Force Office of Scientific Research and David Campbell of ERC, Inc., for sponsoring this research.

\section{References}

[1] Kaufman, H. R., "Technology of Closed-Drift Thrusters," AIAA Journal, Vol. 23, No. 1, 1985, pp. 78-87.

[2] Zhurin, V. V., Kaufman, H. R., and Robinson, R. S., "Physics of Closed Drift Thrusters," Plasma Sources Science and Technology, Vol. 8, Feb. 1999, pp. R1-R20.

[3] Spores, R. A., Spanjers, G. G., Birkan, M., Lawrence, T. J., "Overview of the USAF Electric Propulsion Program," 37th AIAA/ASME/SAE/ ASEE Joint Propulsion Conference, AIAA Paper 2001-3225, 2001.

[4] Spanjers, G. G., Birkan, M. and Lawrence, T. J., "The USAF Electric Propulsion Research Program," 36th AIAA/ASME/SAE/ASEE Joint Propulsion Conference, AIAA Paper 2000-3146, 2000.

[5] Beal, B. E., Gallimore, A. D., and Hargus, W. A., "Preliminary Plume Characterization of a Low-Power Hall Thruster Cluster," 38th AIAA/ ASME/SAE/ASEE Joint Propulsion Conference, AIAA Paper 20024251, 2002.

[6] Hargus, W. A., and Reed, G., "The Air Force Clustered Hall Thruster Program," 38th AIAA/ASME/SAE/ASEE Joint Propulsion Conference, AIAA Paper 2002-3678, 2002.

[7] Beal, B. E., and Gallimore, A. D., "Energy Analysis of a Hall Thruster 
Cluster," 2003 International Electric Propulsion Conference, Electric Rocket Propulsion Society, Santa Fe, NM, IEPC Paper 2003-0035, 2003.

[8] Beal, B. E., "Clustering of Hall Effect Thrusters for High-Power Electric Propulsion Applications," Ph.D. Dissertation, Department of Aerospace Engineering, Univ. of Michigan, Ann Arbor, MI, 2004.

[9] Beal, B. E., Gallimore, A. D., Hargus, W. A., Jr., "Plasma Properties in the Plume of a Hall Thruster Cluster," Journal of Propulsion and Power, Vol. 20, No. 6, Nov.-Dec. 2004, pp. 985-991.

[10] Hruby, V., Monheiser, J., Pote, B., Rostler, P., Kolencik, J., and Freeman, C., "Development of Low-Power Hall Thrusters," 1999 AIAA Plasmadynamics and Lasers Conference, AIAA Paper 99-3534, 1999.

[11] Schott, L., "Electrical Probes," in Plasma Diagnostics, edited by W. Lochte-Holtgreven, American Inst. of Physics, Woodbury, NY, 1995.

[12] Beal, B. E., Gallimore, A. D., and Hargus, Jr., "The Effects of Cathode Configuration on Hall Thruster Cluster Plume Properties," 41st AIAA/ ASME/SAE/ASEE Joint Propulsion Conference, AIAA Paper 20053678, 2005.

[13] Chen, S., and Sekiguchi, T., "Instantaneous Direct-Display System of Plasma Parameters by Means of Triple Probe," Journal of Applied Physics, Vol. 36, 1965, pp. 2363-2375.

[14] Tilley, D. L., Gallimore, A. D., Kelly, A. J., and Jahn, R. G., "The Adverse Effect of Ion Drift Velocity Perpendicular to a Cylindrical Triple Probe," Review of Scientific Instruments, Vol. 65, No. 3, Mar. 1994, pp. 678-681.

[15] Haas, J. M., and Gallimore, A. D., "Internal Plasma Potential Profiles in a Laboratory Model Hall Thruster," Physics of Plasmas, Vol. 8, No. 2, 2001, pp. 652-660.

[16] Kemp, R. F., and Sellen, J. M., Jr., "Plasma Potential Measurements by Electron Emissive Probe," Review of Scientific Instruments, Vol. 37, No. 4, 1966, pp. 455-461.

[17] Ye, M. Y., and Takamura, S., "Effect of Space Charge Limited Emission on Measurements of Plasma Potential Using Emissive Probes," Physics of Plasmas, Vol. 7, No. 8, Aug. 2000, pp. 3457-3463.

[18] Mikellides, I. G., Katz, I., Goebel, D. M., and Polk, J. E., "Theoretical Model of a Hollow Cathode Insert Plasma," 40th AIAA/ASME/SAE/ ASEE Joint Propulsion Conference, AIAA Paper 2004-3817, 2004.

[19] Peterson, P. Y., Gallimore, A. D., and Haas, J. M., "An Experimental Investigation of the Internal Magnetic Field Topography of an Operating Hall Thruster," Physics of Plasmas, Vol. 9, No. 10, 2002, pp. 4354-4362.

[20] Walker, M. L. R., "Effects of Facility Backpressure on the Performance and Plume of a Hall Thruster," Ph.D. Dissertation, Department of Aerospace Engineering, Univ. of Michigan, Ann Arbor, MI, 2005.

[21] Zakharenkov, L. E., Semenkin, A. V., Rusakov, A. V., Urchenko, N. A., Tverdokhlebov, S. O., Garkusha, V. I., Lebedev, U. V., Podkolsin, S. N., and Fife, J. M., "Study of Multi Thruster Assembly Operation," 2003 International Electric Propulsion Conference, Electric Rocket Propulsion Society, Santa Fe, NM, International Electric Propulsion Conference Paper 2003-0311, 2003.

R. Myers Associate Editor

\section{Basic Helicopter Aerodynamics, Second Edition}

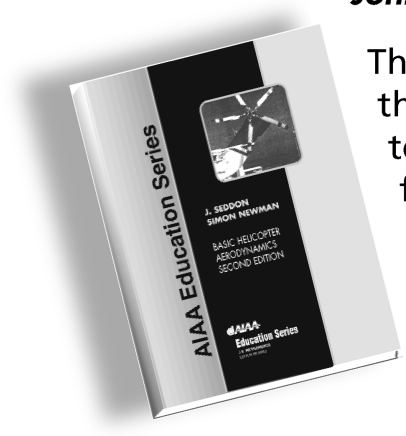

\section{John Seddon and Simon Newman}

This book describes the aerodynamics of helicopter flight, concentrating on the well-known Sikorsky form of single main rotor and tail rotor. Early chapters analyze the aerodynamics of the rotor in hover, vertical flight, forward flight, and climb to the stage of obtaining the principal results for thrust, power, and associated quantities. Later chapters discuss the characteristics of the overall helicopter, its performance, stability, and control.

Aerodynamic research is also discussed with some reference to aerodynamic design practice.

\section{$* *$ Contents ***}

Introduction
Rotor in Vertical Flight:
Momentum Theory and
Wake Analysis
Rotor in Vertical Flight:
Blade Element Theory
Rotor Mechanisms for
Forward Flight

- Introduction

Rotor in Vertical Flight: Momentum Theory and Wake Analysis

Rotor in Vertical Flight:

Rotor Mechanisms for Forward Flight
Rotor Aerodynamics in Forward Flight

- Aerodynamic Design

- Performance

- Trim, Stability, and Control

$>$ Index
Copublished with Blackwell Science Ltd. Outside the United States and Canada, order from Blackwell Science Ltd., United Kingdom, tel 441865206206.

AIAA Education Series 2001, 156 pages, Hardback ISBN: 1-56347-510-3

List Price: $\$ 68.95$

AIAA Member Price: \$49.95
IIIA

American Institute of Aeronautics and Astronautics 